\title{
Peripheral Electrical and Magnetic Stimulation to Augment Resistance Training
}

\author{
Jawad F. Abulhasan *, Yvonne L. D. Rumble, Emma R. Morgan, William H. Slatter and \\ Michael J. Grey \\ School of Sport, Exercise and Rehabilitation Sciences, University of Birmingham, Edgbaston Campus, \\ Birmingham, West Midlands B15 2TT, UK; y.rumble@hotmail.co.uk (Y.L.D.R.); morgan3193@gmail.com (E.R.M.); \\ slatter@hotmail.co.uk (W.H.S.); drmichaeljgrey@gmail.com (M.J.G.) \\ * Correspondence: jawad.abulhasan@gmail.com; Tel.: +965-6666-7770
}

Academic Editors: Giuseppe Musumeci and Paola Castrogiovanni Received: 5 June 2016; Accepted: 30 August 2016; Published: 13 September 2016

\begin{abstract}
Electrical stimulation (ES) and magnetic stimulation (MS), applied peripherally, may be used to elicit muscle contractions to increase muscle hypertrophy, increase muscle strength and reduce knee laxity in rehabilitation following injury. We aimed to examine the effect of a three-week exercise programme designed to induce muscle hypertrophy augmented by peripheral ES and MS. We hypothesised that the use of peripheral stimulation to augment voluntary drive during a resistance-training protocol would induce more repetitions thus leading to increased thigh circumference, muscle layer thickness, and quadriceps strength whilst decreasing knee laxity. Thirty healthy participants were divided randomly into either ES, MS or Control groups. Five resistance training sessions were carried out, consisting of four sets of quadriceps extensions. During the first three sets the participants performed eight repetitions at $85 \%$ of their 1-repetition maximum (1-RM). On the last set, the participants were instructed to perform the exercise until failure. The augmentation of peripheral stimuli allowed the MS and ES groups to continue to exercise producing, on average, $4 \pm 2$ and $7 \pm 6$ additional repetitions with ES and MS, respectively. Following the training, significant increases were observed for both 1-RM $(p=0.005)$ and muscle layer thickness $(p=0.031)$ whilst no change was observed in thigh circumference $(p=0.365)$. Knee laxity decreased $(p=0.005)$. However, there were no significant differences in the stimulation groups compared with control for any of these measurements. The additional repetitions elicited by stimulation after the point of failure suggests that peripheral electrical and/or magnetic stimulation may be useful as an adjunct for resistance training. However, this effect of resistance training augmented by peripheral stimulation on hypertrophy, strength and knee laxity may be small.
\end{abstract}

Keywords: k electrical stimulation; magnetic stimulation; strength

\section{Introduction}

Resistance training is frequently used to promote muscle hypertrophy, strength and knee laxity. Electrical stimulation (ES) and magnetic stimulation (MS) have been used as an adjunct to athletic training [1-3], although it is more commonly used as a rehabilitation therapy to promote quadriceps strength after surgery [4-7], quadriceps hypertrophy [8], to investigate different types of muscle fatigue [9], daily functional activities after stroke [10], and osteoarthritis patients [11,12]. However, the evidence concerning the usefulness of peripheral stimuli as an adjunct to training programmes for enhancing muscle hypertrophy, strength, knee laxity and training healthy people is not conclusive. One reason for the inconsistence effects reported in the literature is the methods by which stimulation is applied. 
Kyung-Min et al. [4] systematically reviewed the literature assessing the effect of ES on quadriceps strength and functional performance following knee surgery. They reported significant effect sizes of quadriceps isometric and isotonic torque (ranging from -0.74 to 3.81) at 6 weeks post-operatively, in contrast, the effect sizes of the lateral step-up test and functional reach test were not significant (ranging from 0.07 to 0.64 ). The authors concluded that ES with exercise may be more effective in enhancing quadriceps strength than exercise alone. In a more recent systematic review, Hewlett et al. [10] examined the ability of ES to improve walking speed, wrist extension and ankle dorsiflexion, and investigated whether it is more effective than training alone. They showed that ES had a mean moderate effect $(0.40,0.09-0.72 ; 95 \% \mathrm{CI})$ on activity compared to no or a placebo intervention. In addition, the stimulation group showed a mean large effect on upper limb activity $(0.69,0.33-1.05 ; 95 \% \mathrm{CI})$ and a small effect on walking speed $(0.08 \mathrm{~m} / \mathrm{s}, 0.02-0.15 ; 95 \% \mathrm{CI})$ compared to the control group. Their findings suggest that ES could be used in patients after traumatic injuries where functions have been affected.

The literature lacks a gold standard anteroposterior knee laxity measure to assess anterior cruciate ligament (ACL) injuries [13]. As a result, clinicians rehabilitate the injured knee through a variety of resistance training protocols with the aim of strengthening thigh muscles to overcome knee instability. Beretta-Piccoli et al. [14] reported that patients showed less fatigability after 24 months of ACL rehabilitation compared to a group of patients who underwent less than 12 months rehabilitation. This suggests that long term resistance training strategies after ACL reconstruction should be implemented to reduce knee injury rates. Taradaj et al. [7] assessed if ACL-reconstructed male football players $(n=40)$ benefited from ES as an adjunct to their regular protocol after knee ACL reconstruction. To the authors' knowledge, this study design had the shortest protocol for their experiment (one month), and both the intervention and control groups received three sessions weekly consisting of the same exercise programme. The intervention group received ES on both right and left quadriceps three times daily, three days a week. The comparison of post-training measures showed a significant difference in favour of the stimulation group in the quadriceps extension $(30.1 \%$ versus $4.6 \%$, $p=0.002)$ and thigh circumference $(1.4 \%$ versus $0.6 \%, p=0.04)$. The authors concluded that there is evidence of the benefit of peripheral ES in restoring quadriceps muscle mass and strength in football players. Barcellona et al. [15] investigated the effect of two sets of 20-RM (LOW group) and 20 sets of 2-RM (HIGH group) quadriceps open kinetic chain resistance training on anterioposterior knee laxity. Unlike the HIGH and control groups, the LOW group demonstrated a mean reduction of $5 \mathrm{~cm}$ in anterior knee laxity after a twelve-week training protocol. The authors concluded that knee extensor open kinetic chain resistance training at the corrected dose may lead to a reduction in anterior knee laxity of the ACL-injured knee. To the author's knowledge, this study is the only one that has investigated the effect of quadriceps hypertrophy training on anterioposterior knee laxity.

In addition to its clinical use, the effect of peripheral stimulation as an adjunct to weight training has been investigated in healthy people. Kubiak et al. [3] compared quadriceps strength torque in control $(n=9)$, isometric exercise $(n=10)$ and ES $(n=10)$ groups before and after a five-week training protocol consisting of three sessions per week. The quadriceps of the stimulation group received 15-s long stimulation contractions with a 50-s rest period between each contraction. All the participants tolerated a stimulation intensity which ranged between $75 \%$ and $134 \%$ of the MVIC, and significant strength increases $(p<0.05)$ were seen for all in both the electrical and isometric exercise groups. Szecsi et al. [1] evaluated the mechanical power generated by healthy participants during MS or ES induced ergometer training conditions; MS produced more mechanical power $(23.8 \pm 9.1 \mathrm{~W})$ and longer cycling exercise compared to ES $(11.3 \pm 11.3 \mathrm{~W})$. Bax et al. [16] systematically reviewed the literature that investigated the effect of $\mathrm{ES}$ as an adjunct to training on the quadriceps femoris muscle strength for both healthy and ACL-reconstructed participants. A number of important conclusions were highlighted in this review. They suggested that the application of ES for both injured and non-injured participants is likely to be more appropriate as an adjunct to rather than a replacement of resistance training. They also suggest voluntary activity together with ES likely results in greater 
efficacy. Their meta-analysis indicated that publication bias may be present in the literature regarding whether the included studies represent the full spectrum of trials performed in actual research practice. Finally, they highlighted the observation that the literature in this field lacks high quality studies and that further research is necessary.

Recently, peripheral MS has been trialled as an alternative to ES [9,17]. As this technique is novel, there is a dearth of literature examining its efficacy as an adjunct to training programmes for quadriceps circumference, muscle layer thickness, strength and knee laxity. Previously, peripheral MS has been restricted to the study of fatigue [18] and it has been reported that peripheral stimulation might minimise the effect of muscle fatigue and shorten the time spent in recovery $[1,7,18]$. In addition, peripheral stimulation alongside weight training may be preferred by patients and athletes as it is characterised by portability (ES) and less pain (MS) compared to alternative methods, such as stretching, massage and cold water immersion [11,19]. Moreover, the application of peripheral ES and MS will bypass central nervous system (CNS) fatigue, and, therefore, it may be more efficacious if applied at the point of voluntary muscle failure in order to induce additional repetitions. Nevertheless, studies that showed a positive effect of peripheral stimulation used subjective outcome measures (e.g., pain), which increase the chances of false positive results [20,21].

We aimed to determine whether peripheral ES and MS applied at the point of muscle failure following voluntary exercise could induce greater hypertrophy, strength and less anterior knee laxity than voluntary muscle activity alone. We hypothesised that a three-week training protocol using peripheral ES or MS applied at the point of voluntary muscle failure would induce more repetitions, and increase thigh circumference, muscle layer thickness, quadriceps strength, whilst decreasing anterior knee laxity compared to the controls.

\section{Materials and Methods}

\subsection{Participants}

Thirty healthy participants ( 16 females, mean age $20 \pm 4$ SD years, range $=18-37$; and 14 males, mean age 19 years $\pm 1 \mathrm{SD}$, range $=18-20$ ) were recruited. All the participants were undergraduate university students who performed active regular exercise of not less than $30 \mathrm{~min}$ of physical activity at least five times per week. Participants were screened for previous knee injuries and neuromuscular conditions, and agreed not to undergo any additional leg strength training during the three weeks of this study. All participants provided written informed consent prior to participating in the study. The experimental procedures were conducted in accordance to the Declaration of Helsinki and were approved by the ethical committee of the University of Birmingham Science, Technology, Engineering and Mathematics (STEM) committee (ethics approval code: ERN-14-0188).

\subsection{Study Design}

The study was carried out over 21 days and had a between-participant design with four dependent variables: girth measurement of the thigh muscle, quadriceps muscle layer thickness, knee anterioposterior laxity measure, and maximum weight lift of the quadriceps extension. Participants were randomly assigned to one of the three study groups: strength training only (control), strength training with electrical stimulation (ES), or strength training with magnetic nerve stimulation (MS). The study had two independent variables: time (pre vs. post) and group (electrical vs. magnetic vs. control). The study protocol started with baseline testing and a weight training session on day one, followed by two rest days, which were also provided between each subsequent training session. After the final training session all the participants rested for a week to ensure no peripheral fatigue existed as a result of the training protocol. Finally, on day 21, post-experimental testing was conducted (Figure 1). All measurements were carried out on the participant's dominant leg in a non-fatigued state. 


\begin{tabular}{|c|c|c|c|c|c|}
\hline $\begin{array}{l}\text { Baseline } \\
\text { measure }\end{array}$ & $\begin{array}{l}\text { All groups } \\
\text { a }\end{array}$ & & Final set & $\begin{array}{l}\text { Extra } \\
\text { reps }\end{array}$ & $\begin{array}{l}\text { Post-training } \\
\text { measure }\end{array}$ \\
\hline \multirow{3}{*}{$\begin{array}{l}\text { 1.Thigh } \\
\text { circumference } \\
\text { 2.Muscle } \\
\text { layer } \\
\text { thickness }\end{array}$} & \multirow{3}{*}{$\begin{array}{l}3 \text { sets } \times 8 \\
\text { reps at } 80 \% \\
\text { of } 1-\mathrm{RM} \\
(30 \text { s rest / } \\
\text { set) }\end{array}$} & \multirow[t]{2}{*}{ Control } & $\begin{array}{l}\text { Training until failure at } \\
80 \% \text { of } 1-\mathrm{RM} \text { counted }\end{array}$ & \multirow[b]{2}{*}{ Counted } & \multirow{3}{*}{$\begin{array}{l}\text { 1.Thigh } \\
\text { circumference } \\
\text { 2. Muscle } \\
\text { layer } \\
\text { thickness } \\
\text { 3. Anterior } \\
\text { knee stability } \\
\text { 4.1-RM }\end{array}$} \\
\hline & & & $\begin{array}{l}\text { Training until failure at } \\
80 \% \text { of } 1-\mathrm{RM}+ \\
\text { individualized electrical } \\
\text { stimulation threshold } \\
\text { applied } \\
\end{array}$ & & \\
\hline & & Magnetic & $\begin{array}{l}\text { Training repetitions } \\
\text { until failure at } 80 \% \text { of } \\
1-\mathrm{RM}+\text { individualized } \\
\text { magnetic stimulation } \\
\text { threshold applied }\end{array}$ & Counted & \\
\hline Day 1 & & & 5 sessions from Day 4 and & every 2 days & Day 21 \\
\hline
\end{tabular}

Figure 1. Flow diagram of the weight training protocol for the three groups. All performed a baseline measure before any weight training followed by four sets of resistance quadriceps weight training. The first three were standardised to eight repetitions only at $80 \%$ of 1-RM. The fourth set was aimed to reach the maximum number of repetition a participant can could perform. For the intervention groups both received either stimulation to assess whether extra repetitions could be induced or not at point of muscle failure. ES = electrical stimulation; $\mathrm{MS}$ = magnetic stimulation; reps = repetitions; 1-RM = 1-repetition maximum.

\subsection{Procedures}

Baseline and post-exercise measures for thigh circumference (Section 2.4.1), muscle layer thickness (Section 2.4.2) and knee laxity (Section 2.4.4) were recorded with the participant laying supine on a plinth. The baseline and post-exercise 1-RM assessment (Section 2.4.3) and fatiguing exercises were conducted with the participant seated in Cybex chair (Cybex VR3, International Inc., Owatonna, MA, USA).

The study training protocol was designed to focus on hypertrophy rather than strength. Previous research has suggested that optimum hypertrophy gains in healthy individuals are best obtained when performing the 1-repetition maximum (1-RM) technique (see [22] for review), although it has also been shown that training with higher intensities can also lead to strength gains in addition to hypertrophy (e.g., [23,24]). Each participant performed three sets of 8 repetitions at $80 \%$ of their 1-RM (as defined in Section 2.4.3) with $30 \mathrm{~s}$ rest between each set (Figure 1). This was followed by a fourth set, also performed at $80 \%$ of the 1-RM, where the participant exercised to the point of muscle failure. Failure was defined as the point at which a participant could no longer voluntarily contract the quadriceps to fully extend the leg. At this point either electrical (ES group) or magnetic (MS group) stimuli were provided to augment the voluntary effort thus allowing the participants to perform additional repetitions beyond the point of failure. In both cases, stimuli were delivered to the motor point of the rectus femoris muscle. Electrical stimuli were delivered through self-adhesive surface stimulating electrodes (Compex Easy Snap, Compex Global, Surrey, UK) using the Mi Compex 3, Professional (Compex Global), with a pulse duration of $400 \mu$ s and a pulse frequency of $50 \mathrm{~Hz}$. To determine the magnitude of the stimuli, the intensity of stimulation was increased to the point where the greatest contraction was produced within the individual tolerance level of the participant. This intensity was recorded as their maximum threshold. The magnitude for magnetic stimuli (MagPro $\times 100$, MagVenture, Farum, Denmark) was determined in a similar manner increasing the stimulation from $5 \%$ intensity until reaching their maximum threshold. 


\subsection{Assessment Methods}

All testing and weight training sessions were supervised by a certified strength and conditioning coach. Post-testing was carried out by an experimenter who had remained blinded throughout the data collection sessions to avoid bias when reading the follow up results. Post-training measurements were carried out at roughly the same time of day $( \pm 2 \mathrm{~h})$ to reduce the effect of circadian fluctuations $[25,26]$. All measures were taken from the participant dominant leg.

\subsubsection{Thigh Circumference}

Thigh circumference was measured with the participant lying supine position on a plinth. The measurement was taken at the midpoint between the anterior superior iliac spine (ASIS) and the lateral epicondyle of the femur, and the position was marked with a permanent marker. Three measures of thigh circumference were made with a medical tape recording to the nearest millimetre, from which a median value was calculated for use in the statistical analysis.

\subsubsection{Muscle Layer Thickness}

With the participant in the same position as for the circumference measure, rectus femoris (RF) muscle layer thickness (MLT) was obtained using a Phillip Sonos D2 5500 ultrasound (US) with an 11-3L probe at an image depth of $7 \mathrm{~cm}$. Measurements were made using the ultrasound's calliper function. Rectus femoris MLT measures were repeated three times to the nearest millimetre, from which a median value was calculated for use in the statistical analysis (Figure 2).

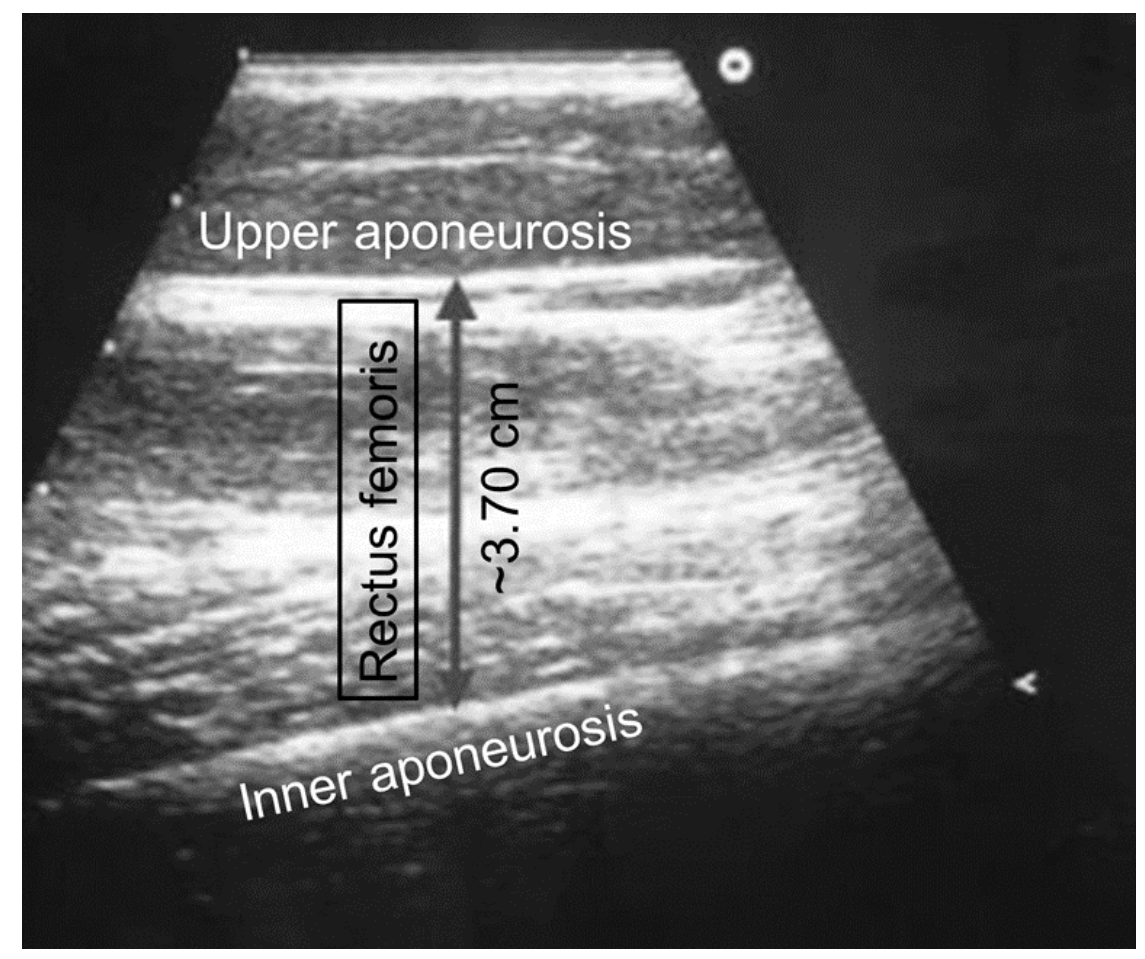

Figure 2. Illustration of how the muscle layer thickens was measured using the ultrasound image for every participant. The distance between the upper layer and lower layer of the rectus femoris muscle image was measured using the integrated US arrow. The US gives the exact distance between the two heads of the arrow which corresponds to the thickness of the measured muscle.

\subsubsection{Maximal Leg Extension}

Knee extension strength was measured with a Cybex VR3 (Figure 3). The participant was seated with the back support and tibia pad adjusted to fit the individual's height, and these seating 
adjustments were recorded in order to replicate the participant's position in all tests. Each participant sat with the hips straight and in line with each other, knees at 90 degrees, the back in a comfortable position and toes dorsiflexed. Following a short warm-up, participants were instructed to perform two repetitions at increasing weight and were challenged with ensuring that their legs reached full extension in a controlled manner. Once the participant could not complete as repetition, this weight was recorded as their one-repetition maximum (1-RM).

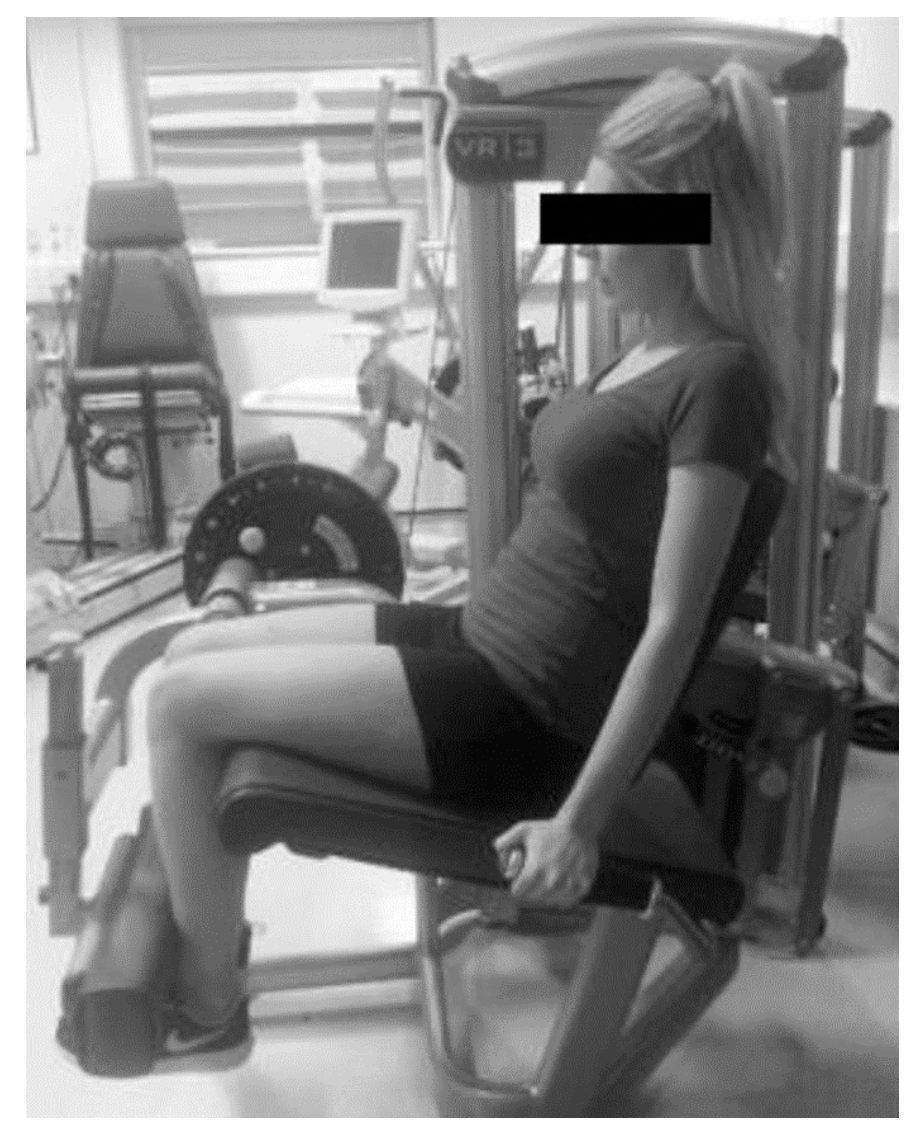

Figure 3. Voluntary leg extension setup and participant in the starting position of the weight training programme. The participant was instructed to fully extend their lower limbs with a slow and controlled movement and then return to the starting point. This cycle was counted as one successfully completed repetition.

\subsubsection{Knee Anterioposterior Laxity Test}

A KT-2000 knee arthrometer (MEDmetric Corp., San Diego, CA, USA) was used to measure the anteroposterior displacement of the femur on the tibia. For this measure the participant remained relaxed in a supine position on the plinth with their dominant knee supported at $30^{\circ}$ of flexion as measured using a goniometer. Initially, the KT-2000 device was secured over the participant's leg in the ideal position with reference to the knee joint line. Both knees were supported on a firm, comfortable platform placed proximal to the popliteal space. In addition, a foot support, supplied in the KT-2000 arthrometer kit, was used to position the leg symmetrically and to avoid external rotation of the tibia. Next, the Lachman test, forced anterior displacement of the tibia with respect to the femur, was performed by holding the femur and pulling on the handle of the KT-2000 (Figure 4). Anterior displacement was measured to the nearest millimetre. Three trials were conducted, with the median calculated for use in the statistical analysis. 


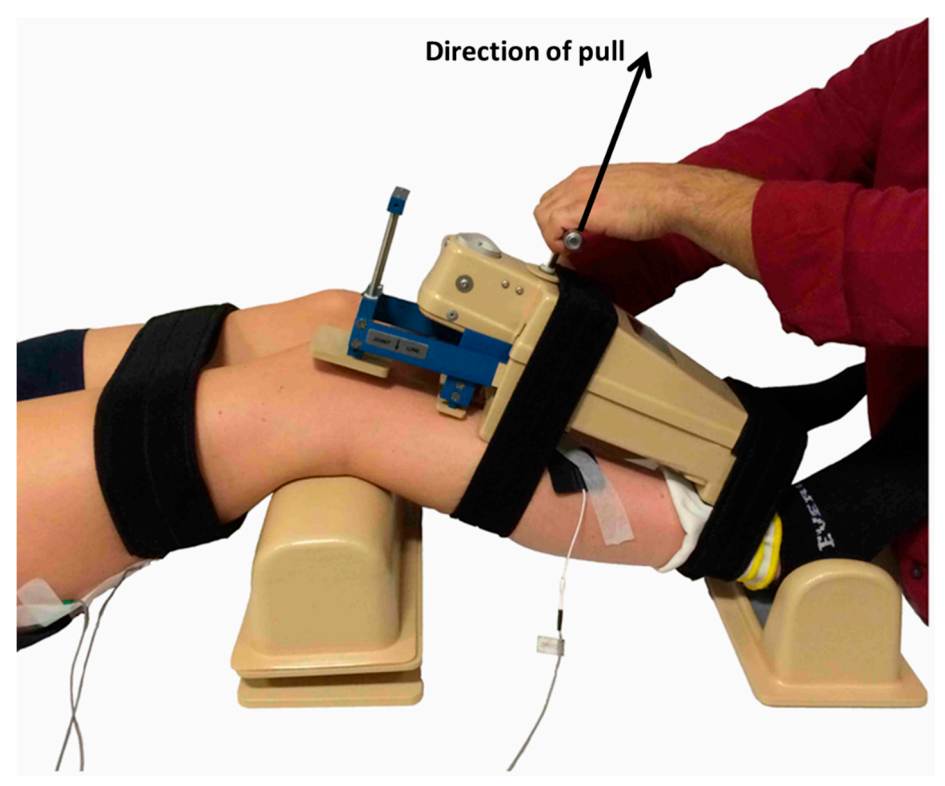

Figure 4. The experimental procedure of the KT-2000 arthrometer and the position of the knee joint, placement of the examiner hands and the direction of the tibial pull is illustrated.

\subsection{Statistical Analysis}

An independent $t$-test was used to compare the additional number of repetitions elicited with ES or MS augmentation after voluntary failure. One-way analyses of variance (ANOVA) tests were used to compare the number of repetitions induced in each group during the final set before stimulation. In addition, two-way (ANOVA) tests were used to examine the time effect (pre and post) and the group effects in the three different study groups (control, ES and MS) for each of the four dependent variables. The normality of the data was assessed using the Kolmogorov-Smirnov test and there were equal variances of the dependent variables, which were assessed using the Levene's test, across all levels of the independent variables. The level of significance was set at $p \leq 0.05$ for all measures. If significance was achieved, then a Tukey post-hoc test was planned to be performed. All the statistical analyses of the data were executed using SPSS Statistics 22 Software (IBM, New York, NY, USA).

\section{Results}

All participants completed the study successfully and tolerated the stimulation well. At the end of the three-week training protocol, the maximum number of repetitions in the final set was $8 \pm 2$, $9 \pm 1$ and $10 \pm 1$ for the control, ES and MS groups, respectively. A one-way ANOVA indicated that there was no significant difference in the mean number of repetitions during the final set between the groups $(p=0.538)$ (Figure 5).

All the participants exposed to stimulation were able to complete addition repetitions; ES $=4 \pm 2$ and MS $=7 \pm 6$, range (1-20). No significant difference between the number of additional repetitions for the ES and MS groups were observed ( $p=0.187$ ) (Figure 5). Participants in the ES group showed more confidence and comfort than the MS group during stimulation. This may be because the ES group had the ability to self-control the intensity of the stimulation as opposed to the MS group, were the intensity of the stimulation was controlled by the experimenter.

Following the training, significant time-effect increases were observed for both 1RM $(p=0.005)$ and muscle layer thickness $(p=0.031)$, whilst no change in thigh circumference $(p=0.365)$ was noted, and knee laxity was observed to decrease $(p=0.005)$. However, there were no group-effect changes for the stimulation groups compared to the control group for any of the measurements: 1-RM $\mathrm{F}(2,27)=0.90, p=0.415$, partial $\eta^{2}=0.03, \mathrm{ICC}=0.60$, statistical power $=0.20$ (Figure $6 \mathrm{c}$ ), muscle layer thickness $\mathrm{F}(2,27)=0.34, p=0.712$, partial $\eta^{2}=0.01, \mathrm{ICC}=0.73$, statistical power $=0.10$ (Figure $6 \mathrm{~b}$ ), thigh 
circumference $\mathrm{F}(2,27)=2.10, p=0.132$, partial $\eta^{2}=0.07$, ICC $=0.75$, statistical power $=0.41$ (Figure 6a), and anterior knee laxity, $\mathrm{F}(2,27)=1.23, p=0.300$, partial $\eta^{2}=0.04$, ICC $=0.35$, statistical power $=0.25$ (Figure 6d).

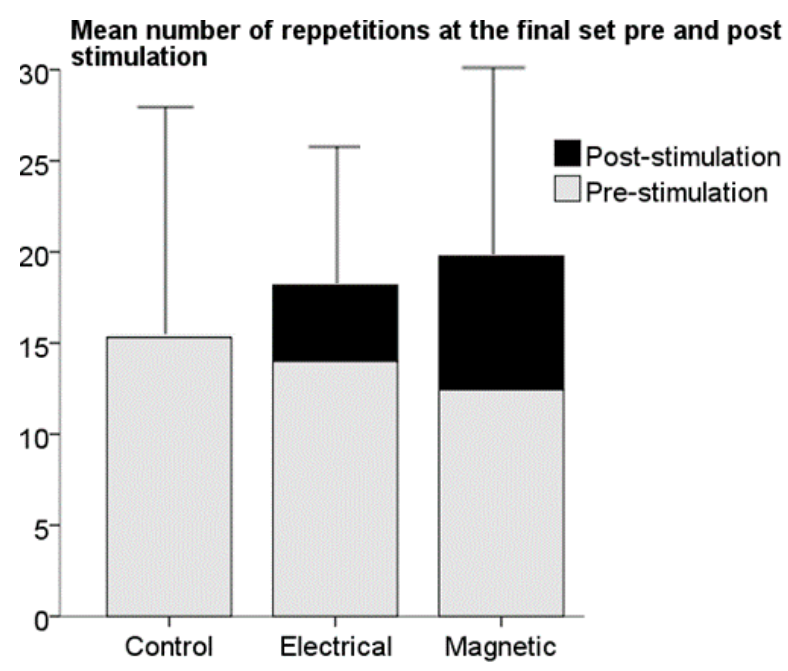

Figure 5. Bar plot of the mean number of repetitions each group performed during the final set before the addition of stimulation at the point of failure and the extra number of repetitions induced after muscle failure had been reached during the last training set in the two intervention groups. No significant difference in the mean of extra repetition numbers was observed between the two intervention groups. Data are expressed as mean \pm standard deviation. $\mathrm{ES}=$ Electrical stimulation group, MS = magnetic stimulation group.
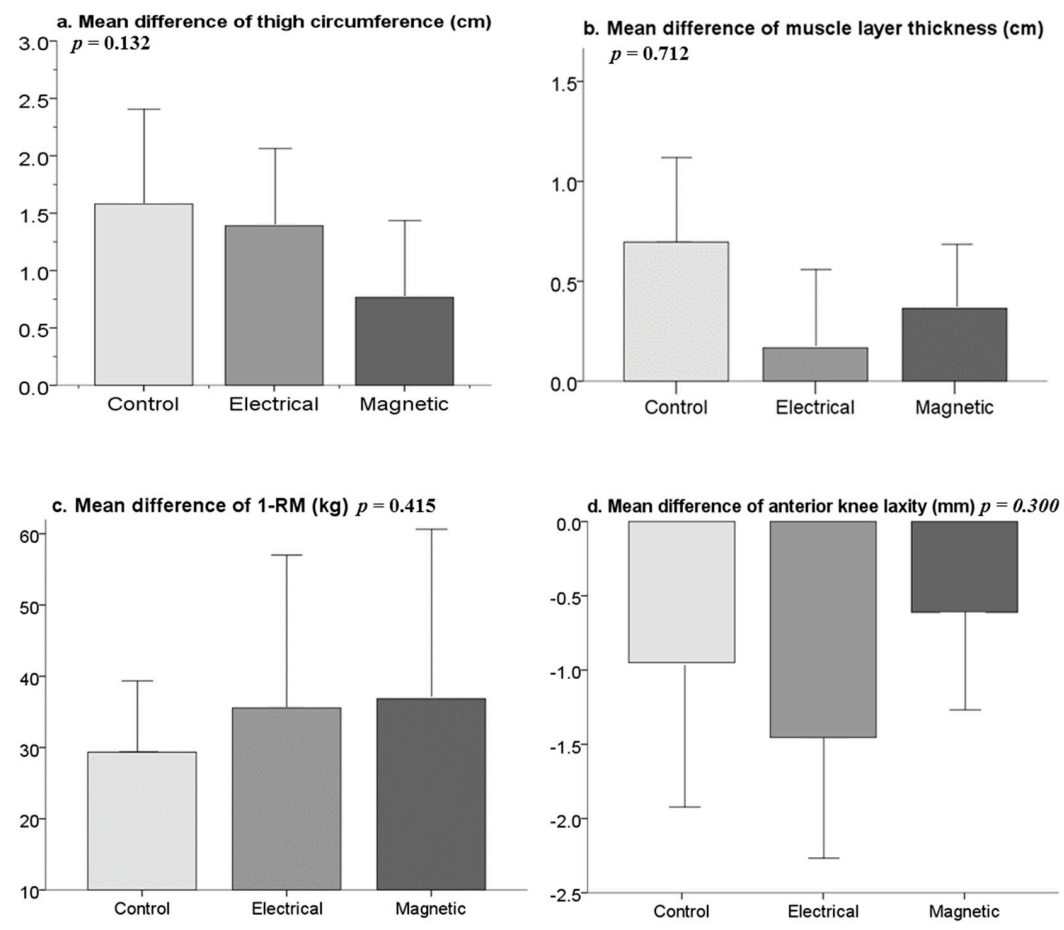

Figure 6. Bar plots of the means of the difference between baseline and post-training measures for all three groups for (a) thigh circumference; (b) muscle layer thickness; (c) 1-repetition maximum and (d) anterioposterior knee laxity. No significant difference was observed between the three groups. Data are expressed as mean \pm standard deviation. 


\section{Discussion}

The aim of this study was to determine if peripheral ES or MS applied at the point of muscle failure following voluntary exercise could induce greater hypertrophy, strength and lesser anterior knee laxity than voluntary muscle activity alone. Both peripheral ES and MS elicited additional repetitions following voluntary muscle failure. However, as applied in the present study, the effect of such training on muscle hypertrophy, strength and anterior knee laxity was not significantly different from voluntary training alone; hence the effect may be small.

The literature describes a variety of approaches that have been used to evaluate muscle strength, such as hand-held dynamometer, isokinetic machines, 1-RM and maximal voluntary contraction technique. There is a consensus in the literature that the 1-RM is a reliable and relatively cost-effective technique, as it requires non-laboratory equipment and consequently is considered one of the most commonly used techniques to assess muscle strength in non-laboratory situations [27-31]; therefore, this measure was used to assess quadriceps muscle strength in this study. The number of extra repetitions for each participant in the final set was widespread (range, ES; 2-8; MS =1-20). Despite the fact that a firm procedure was used, in this study, to establish a participant's 1-RM, several factors may have affected the outcome and there remains the possibility that the participants who executed a higher number of repetitions had not in fact exercised at their 1-RM. This may be due to both neural and psychological supraspinal drive inhibition operating on the muscle motor units [32]. Belanger and McComas [33] reported that 50\% of participants did not reach their 1-RM even when they were asked to exert force with their maximal volition. Therefore, this study shows that our developed 1-RM technique can better ensure that maximum weight is reached. This could be achieved by overtaking the neural inhibition through the use of peripheral stimulation. Thus, the results of this study show that the use of ES and MS alongside the traditional 1-RM technique can help better predict the actual 1-RM for the quadriceps muscle. This novel technique could be a useful tool to accurately measure the maximum voluntary contraction of patients after injury and/or athletes during training. Thus, our novel technique could help guide the return-to-play decision as maximum strength and hypertrophy is warranted for safe return-to-play [23,34,35].

Although the effect of weight training on muscle hypertrophy has been investigated in numerous studies, there is no information, to our knowledge, regarding the effect of ES and MS applied at the point of muscle failure following voluntary exercise on hypertrophy. Even though previous investigations have used ES or MS as standalone study arms to enhance hypertrophy [36-38], no study has investigated if stimulation applied at the point of muscle failure following voluntary exercise could induce greater hypertrophic changes than voluntary muscle activity alone. Our results indicate that there was no significant hypertrophic difference in the thigh circumference $F(2,27)=2.10$, $p=0.132$, partial $\eta^{2}=0.01$ (Figure 6a) or muscle layer thickness $\mathrm{F}(2,27)=0.34, p=0.712$, partial $\eta^{2}=0.01$ (Figure $6 \mathrm{~b}$ ) following the application of ES or MS between the study groups. This may be due to fat loss [39] or non-hypertrophic adaptation of the neuromuscular system in response to static resistance training [40], rather than a change within the quadriceps circumference. In line with the above, Carolan and Cafarelli, [41] reported that after the first week of their training protocol the quadriceps extension antagonist muscle (hamstring) showed a decrease in muscle co-activation by $20 \%$. This non-hypertrophic adaptation of the neuromuscular system resulted in a reduction of thigh circumference post-training.

The results of the presented study showed no significant difference in strength, although there was a trend for the stimulated groups to be greater than the controls (Figure 6). In addition, our results showed a small trend towards a decrease in muscle size as determined by thigh circumference (Figure 6a) and muscle layer thickness (Figure 6b). This is seemingly paradoxical, but Brook et al. [42] in his recent review concluded that the adaptations of muscle mass and strength to resistance-type training are as yet unclear. Previous literature has shown inconsistent results in the effect of resistance-type exercises on muscle mass and strength. For example, Farup et al. [43] reported a significant increase in both quadriceps muscle mass and strength [42-44] following a 10-week resistance training programme. 
In contrast, McBride et al. [45] reported a significant gain in quadriceps strength with no gain in lean muscle mass following a 12-week programme of leg press resistance training. The controversy in the literature regarding the relationship between strength and hypertrophy gains after resistance training may be explained by the insensitivity of the muscle lean mass measures and differences in muscle-fibre types [46]. Magnetic resonance scanning, dual-energy X-ray absorptiometry (DXA), and computed tomography (CT) are considered the best measures of muscle mass $[47,48]$ but they are not cost-effective. US is a commonly used, cost-effective, portable and quick device with which to quantify hypertrophy [49-51]. Unlike [43,52,53] who used DXA, MRI and CT, respectively, as a measure of lean muscle mass and reported significant hypertrophic changes, we used US to measure hypertrophic changes. It is crucial to shed light on the notion that the existing literature on "changes" in lean muscle mass in response to resistance training depends upon the method chosen to detect muscle hypertrophy [54]. Hence, experience of the principle investigator in performing the US procedure and the insensitivity of the US device may have played a role in the outcome of our study. Consequently, more studies are needed in order to confirm if peripheral ES and MS applied at the point of muscle failure following voluntary exercise can induce significant hypertrophic changes more than voluntary muscle activity alone in terms of the use of sensitive measures of hypertrophic muscle changes.

Although the baseline measures differed between participants in the three groups, the tendency to a positive change of quadriceps strength as measured by the 1-RM (Figure 6c) in all groups is promising, despite the non-significant difference between the groups (CONT $=29.30 \pm 10 \mathrm{~kg}$, ES $=35.55 \pm 21.38 \mathrm{~kg}$, MS $=36.85 \pm 23.61 \mathrm{~kg}$ ). Previous studies have found a significant effect of ES on muscle strength versus a control group [7], and over periods as short as five weeks [2,3,12]. However, no studies have investigated if peripheral ES and MS applied at the point of muscle failure following voluntary exercise could induce greater strength changes than voluntary muscle activity alone. Hence, it is worth emphasising that the present study showed that the positive change in the ES and MS groups were bigger than in the control group. The findings of this study suggest that regardless of the non-significant results, a direct comparison between the two experimental groups revealed that MS induced more repetitions $(7 \pm 6)$ than ES $(4 \pm 2)$. Consequently, extra repetitions at 1-RM means extra strength gain [55]. Thus, the use of this novel protocol in traumatised patients will perhaps reduce quadriceps wasting in the early stages of return-to-play rehabilitation programmes.

There is a consensus in the literature that strength training enhances knee stability [34,56-59]. However, the present study showed mixed results in anterioposterior knee laxity measurements. Although the majority of the participants (25/30) showed a reduction in their anterior laxity post-training, some (5/30) demonstrated an increase. A possible justification as to why anterioposterior knee laxity had increased after quadriceps weight training is the relaxed state of the hamstrings muscles during the anterioposterior laxity measurement. Several studies have shown that lateral hamstring activity during the knee laxity test reduces anterioposterior laxity $[15,60,61]$. In contrast, in the present study the procedure for the knee laxity test adhered to the guidelines for use of the KT-2000 arthrometer [62], and the participants ensured that their hamstrings remained fully relaxed during the knee laxity testing. Barcellona et al. [15] reported a non-linear relationship between hamstrings muscle activity and anterioposterior knee laxity measured by the KT-2000 for a maximal manual technique. They reported that $2 \%$ of EMG activity of the lateral hamstring caused an approximately $45 \%$ laxity reduction in anterioposterior laxity. Moreover, Markolf et al. [60] reported that contraction of the hamstrings muscles reduces knee laxity to $25 \%-50 \%$ of the normal values.

To summarise, there is controversy in the literature as to whether the hamstrings muscles should be activated or should remain fully relaxed during an anterioposterior knee laxity test. The aim of the test could determine which method is recommended, as an anterioposterior knee laxity test with relaxed hamstrings shows the displacement of the tibia on the femur in a static state, while conducting a knee laxity test with activation of the hamstrings correlates better with the actual status of the knee during exercise. Therefore, questions arise regarding which test condition replicates the actual kinematics and kinetics of the knee. This debate may be better directed towards a more robust 
test that can replicate the dynamic condition of the knee during exercise. Therefor this favours the argument that knee laxity is better measured through activation of the hamstrings in order to replicate the actual condition of the knee during sport participation. Additionally, whilst there is an agreement in the literature that the ACL is one of the most injured ligament in the knee forcing long absences by athletes [63-66], future work might be directed towards monitoring hamstrings activity during an anterioposterior knee laxity test in order to potentially improve the chances of detecting a possible ACL injury as both knee stability (primary) alongside muscle activity around the knee (secondary) are required for knee stability.

\section{Conclusions}

Whilst this was a small study $(n=30)$, both peripheral stimulation interventions suggested a beneficial trend during strength training by allowing the production of additional repetitions at the point of muscle failure, when it was not possible to continue with voluntary activation alone. In the present study peripherally applied electrical and magnetic stimulation produce similar results, but the electrical stimulation was considered more tolerable than the magnetic stimulation. Furthermore, from a practical point of view electrical stimulation is considerably less expensive with most commercial stimulators having the capability of deliver stimuli simultaneously over two or more channels compared with the single coil for magnetic stimulation.

In the clinic, the use of such peripheral stimuli may be beneficial to offset muscle atrophy, facilitate early rehabilitation, and enhance resistance training protocols, especially when used for patients requiring rehabilitation associated with ACL reconstruction. Specifically, traumatised patients and athletes who aim for a faster return-to-play might benefit from the use of peripheral stimulation as an adjunct to weight training. Exactly how robust peripheral stimulation applied at the point of muscle failure following voluntary exercise has an effect on quadriceps strength, hypertrophy and knee stability warrants further investigation.

Acknowledgments: This article presents independent research funded by the National Institute for Health research (NIHR) Surgical Reconstruction and Microbiology Research Centre (partnership between University Hospitals Birmingham NHS Foundation Trust, the University of Birmingham and the Royal Centre for Defence Medicine). The views expressed are those of the authors and not necessarily those of the NHS, the NIHR or the Department of Health. The research is also funded by the Medical Research Council and Arthritis Research UK as part of the MRC-ARUK Centre for Musculoskeletal Ageing Research (Grant Reference Number: MR/K00414X/1). JA is funded by a scholarship obtained from the Cultural Office at the Kuwait Embassy in London. No other financial assistance was received for this project.

Author Contributions: Jawad F. Abulhasan wrote the manuscript and responded to all the feedback and comments from the co-authors. Jawad F. Abualhasan, Yvonne L. D. Rumble and Emma R. Morgan jointly performed all the tests. William H. Slatter supervisor all the training sessions in the notion of health and safety. William H. Slatter revisited the manuscript for comments and feedback. Michael J. Grey supervised the study, provided feedback and comments on every draft of the manuscript and hosted all the laboratory work inside his laboratory at the University of Birmingham.

Conflicts of Interest: The authors declare no conflict of interest. The founding sponsors had no role in the design of the study; in the collection, analyses, or interpretation of data; in the writing of the manuscript, and in the decision to publish the results.

\section{References}

1. Szecsi, J.; Straube, A.; Fornusek, C. Comparison of the pedalling performance induced by magnetic and electrical stimulation cycle ergometry in able-bodied subjects. Med. Eng. Phys. 2014, 36, 484-489. [CrossRef] [PubMed]

2. Laughman, R.K.; Youdas, J.W.; Garrett, T.R.; Chao, E.Y. Strength changes in the normal quadriceps femoris muscle as a result of electrical stimulation. Phys. Ther. 1983, 63, 494-499. [PubMed]

3. Kubiak, R.J., Jr.; Whitman, K.M.; Johnston, R.M. Changes in quadriceps femoris muscle strength using isometric exercise versus electrical stimulation. J. Orthop. Sports Phys. Ther. 1987, 8, 537-541. [CrossRef] [PubMed] 
4. Kyung-Min Kim, T.C.; Jay, H.; Susan, S. Effects of neuromuscular electrical stimulation after anterior cruciate ligament reconstruction on quadriceps strength, function, and patient-oriented outcomes: A systematic review. J. Orthop. Sports Phys. Ther. 2010, 40, 383-391.

5. Imoto, A.M.; Peccin, S.; Almeida, G.J.M.; Saconato, H.; Atallah, Á.N. Effectiveness of electrical stimulation on rehabilitation after ligament and meniscal injuries: A systematic review. Sao Paulo Med. J. 2011, 129, 414-423. [PubMed]

6. Draper, V.; Ballard, L. Electrical stimulation versus electromyographic biofeedback in the recovery of quadriceps femoris muscle function following anterior cruciate ligament surgery. Phys. Ther. 1991, 71, 455-461. [PubMed]

7. Taradaj, J.; Halski, T.; Kucharzewski, M.; Walewicz, K.; Smykla, A.; Ozon, M.; Slupska, L.; Dymarek, R.; Ptaszkowski, K.; Rajfur, J. The effect of neuromuscular electrical stimulation on quadriceps strength and knee function in professional soccer players: Return to sport after acl reconstruction. BioMed. Res. Int. 2013, 2013. [CrossRef] [PubMed]

8. Barcellona, M.; Morrissey, M.; Milligan, P.; Clinton, M.; Amis, A. The effect of knee extensor open kinetic chain resistance training in the acl-injured knee. Knee Surg. Sports Traumatol. Arthrosc. 2015, 23, 3168-3177. [CrossRef] [PubMed]

9. Millet, G.Y.; Bachasson, D.; Temesi, J.; Wuyam, B.; Féasson, L.; Vergès, S.; Lévy, P. Potential interests and limits of magnetic and electrical stimulation techniques to assess neuromuscular fatigue. Neuromuscul. Disord. 2012, 22 (Suppl. S3), S181-S186. [CrossRef] [PubMed]

10. Howlett, O.A.; Lannin, N.A.; Ada, L.; McKinstry, C. Functional electrical stimulation improves activity after stroke: A systematic review with meta-analysis. Arch. Phys. Med. Rehabil. 2015, 96, 934-943. [CrossRef] [PubMed]

11. Negm, A.; Lorbergs, A.; MacIntyre, N.J. Efficacy of low frequency pulsed subsensory threshold electrical stimulation vs. placebo on pain and physical function in people with knee osteoarthritis: Systematic review with meta-analysis. Osteoarthr. Cartil. 2013, 21, 1281-1289. [CrossRef] [PubMed]

12. Hasan, S. Quadriceps femoris strength training: Effect of neuromuscular electrical stimulation vs. isometric exercise in osteoarthritis of knee. Indian J. Physiother. Occup. Ther. Int. J. 2015, 9, 129-134. [CrossRef]

13. Abulhasan, J.F.; Snow, M.D.; Anley, C.M.; Bakhsh, M.M.; Grey, M.J. An extensive evaluation of different knee stability assessment measures: A systematic review. J. Funct. Morphol. Kinesiol. 2016, 1, 209-229. [CrossRef]

14. Beretta-Piccoli, M.; Schneebeli, A.; Egloff, M.; Cescon, C.; Clijsen, R.; Togninalli, D.; Barbero, M. Myoelectric manifestations of fatigue after acl reconstruction: A cross-sectional study after postoperative rehabilitation. J. Funct. Morphol. Kinesiol. 2016, 1, 193-199. [CrossRef]

15. Barcellona, M.; Morrissey, M.; Milligan, P.; Amis, A. The effect of thigh muscle activity on anterior knee laxity in the uninjured and anterior cruciate ligament-injured knee. Knee Surg. Sports Traumatol. Arthrosc. 2014, 22, 2821-2829. [CrossRef] [PubMed]

16. Bax, L.; Staes, F.; Verhagen, A. Does neuromuscular electrical stimulation strengthen the quadriceps femoris? Sports Med. 2005, 35, 191-212. [CrossRef] [PubMed]

17. Atzori, T.; Vettori, A.; Coli, C.; Gagliardi, E.; Innocenti Bruni, G.; Carrai, R.; Gigliotti, F.; Grippo, A. Muscle repetitive magnetic stimulation in the rehabilitation protocol: Assessment of feasibility and efficacy in normal subjects and in patients with muscle disease. Clin. Neurophysiol. 2013, 124, e205. [CrossRef]

18. Kim, S.-B.; Kim, J.-Y.; Park, S.-W.; Lee, N.-R.; Kim, Y.-H.; Lee, K.-J.; Lee, Y.-H. Effects of pemfs (pulsed electromagnetic fields) stimulation on acupoint in quadriceps fatigue recovery. Int. J. Precis. Eng. Manuf. 2012, 13, 1697-1703. [CrossRef]

19. Thakral, G.; Kim, P.J.; LaFontaine, J.; Menzies, R.; Najafi, B.; Lavery, L.A. Electrical stimulation as an adjunctive treatment of painful and sensory diabetic neuropathy. J. Diabetes Sci. Technol. 2013, 7, 1202-1209. [CrossRef] [PubMed]

20. Doix, A.-C.M.; Matkowski, B.; Martin, A.; Roeleveld, K.; Colson, S.S. Effect of neuromuscular electrical stimulation intensity over the tibial nerve trunk on triceps surae muscle fatigue. Eur. J. Appl. Physiol. 2014, 114, 317-329. [CrossRef] [PubMed]

21. Green, S.; Robinson, E.; Wallis, E. Assessment of calf muscle fatigue during submaximal exercise using transcranial magnetic stimulation versus transcutaneous motor nerve stimulation. Eur. J. Appl. Physiol. 2014, 114, 113-121. [CrossRef] [PubMed] 
22. McMaster, D.T.; Gill, N.; Cronin, J.; McGuigan, M. A brief review of strength and ballistic assessment methodologies in sport. Sports Med. 2014, 44, 603-623. [CrossRef] [PubMed]

23. Peterson, M.D.; Rhea, M.R.; Alvar, B.A. Maximizing strength development in athletes: A meta-analysis to determine the dose-response relationship. J. Strength Cond. Res. 2004, 18, 377-382. [CrossRef] [PubMed]

24. Thornton, M.K.; Potteiger, J.A. Effects of resistance exercise bouts of different intensities but equal work on epoc. Med. Sci. Sports Exerc. 2002, 34, 715-722. [CrossRef] [PubMed]

25. Kong, T.Q.; Goldberger, J.J.; Parker, M.; Wang, T.; Kadish, A.H. Circadian variation in human ventricular refractoriness. Circulation 1995, 92, 1507-1516. [CrossRef] [PubMed]

26. Coldwells, A.; Atkinson, G.; Reilly, T. Sources of variation in back and leg dynamometry. Ergonomics 1994, 37, 79-86. [CrossRef] [PubMed]

27. Phillips, W.T.; Batterham, A.M.; Valenzuela, J.E.; Burkett, L.N. Reliability of maximal strength testing in older adults1. Arch. Phys. Med. Rehabil. 2004, 85, 329-334. [CrossRef] [PubMed]

28. Levinger, I.; Goodman, C.; Hare, D.L.; Jerums, G.; Toia, D.; Selig, S. The reliability of the 1rm strength test for untrained middle-aged individuals. J. Sci. Med. Sport 2009, 12, 310-316. [CrossRef] [PubMed]

29. Abdul-Hameed, U.; Rangra, P.; Shareef, M.Y.; Hussain, M.E. Reliability of 1-repetition maximum estimation for upper and lower body muscular strength measurement in untrained middle aged type 2 diabetic patients. Asian J. Sports Med. 2012, 3, 267-273. [CrossRef] [PubMed]

30. Kraemer, W.J.; Fry, A.; Ratamess, N.; French, D. Strength testing: Development and evaluation of methodology. Physiol. Assess. Hum. Fit. 1995, 2, 119-150.

31. Fleck, S.J.; Kraemer, W. Designing Resistance Training Programs, 4th ed.; Human Kinetics: Champaign, IL, USA, 2014.

32. Ikai, M.; Steinhaus, A.H. Some factors modifying the expression of human strength. J. Appl. Physiol. 1961, 16, 157-163. [PubMed]

33. Belanger, A.; McComas, A.T. Extent of motor unit activation during effort. J. Appl. Physiol. 1981, 51, 1131-1135. [PubMed]

34. Cinar-Medeni, O.; Baltaci, G.; Bayramlar, K.; Yanmis, I. Core stability, knee muscle strength, and anterior translation are correlated with postural stability in anterior cruciate ligament-reconstructed patients. Am. J. Phys. Med. Rehabil. 2015, 94, 280-287. [CrossRef] [PubMed]

35. Palmieri-Smith, R.M.; Lepley, L.K. Quadriceps strength asymmetry after anterior cruciate ligament reconstruction alters knee joint biomechanics and functional performance at time of return to activity. Am. J. Sports Med. 2015, 43, 1662-1669. [CrossRef] [PubMed]

36. Gorgey, A.; Khalil, R. Neuromuscular electrical stimulation training increases intermuscular fascial length but not tendon cross-sectional area after spinal cord injury. Top. Spinal Cord Inj. Rehabil. 2015, 21, 87-92. [CrossRef] [PubMed]

37. Sillen, M.J.H.; Franssen, F.M.E.; Gosker, H.R.; Wouters, E.F.M.; Spruit, M.A. Metabolic and structural changes in lower-limb skeletal muscle following neuromuscular electrical stimulation: A systematic review. PLoS ONE 2013, 8, e69391. [CrossRef] [PubMed]

38. Hasegawa, S.; Kobayashi, M.; Arai, R.; Tamaki, A.; Nakamura, T.; Moritani, T. Effect of early implementation of electrical muscle stimulation to prevent muscle atrophy and weakness in patients after anterior cruciate ligament reconstruction. J. Electromyogr. Kinesiol. 2011, 21, 622-630. [CrossRef] [PubMed]

39. Tremblay, A.; Després, J.P.; Leblanc, C.; Craig, C.L.; Ferris, B.; Stephens, T.; Bouchard, C. Effect of intensity of physical activity on body fatness and fat distribution. Am. J. Clin. Nutr. 1990, 51, 153-157. [PubMed]

40. Billot, M.; Duclay, J.; Simoneau-buessinger, E.M.; Ballay, Y.; Martin, A. Is co-contraction responsible for the decline in maximal knee joint torque in older males? Age 2014, 36, 899-910. [CrossRef] [PubMed]

41. Carolan, B.; Cafarelli, E. Adaptations in coactivation after isometric resistance training. J. Appl. Physiol. 1992, 73, 911-917. [PubMed]

42. Brook, M.S.; Wilkinson, D.J.; Mitchell, W.K.; Lund, J.N.; Szewczyk, N.J.; Greenhaff, P.L.; Smith, K.; Atherton, P.J. Skeletal muscle hypertrophy adaptations predominate in the early stages of resistance exercise training, matching deuterium oxide-derived measures of muscle protein synthesis and mechanistic target of rapamycin complex 1 signaling. FASEB J. 2015, 29, 4485-4496. [CrossRef] [PubMed]

43. Farup, J.; Kjølhede, T.; Sørensen, H.; Dalgas, U.; Møller, A.B.; Vestergaard, P.F.; Ringgaard, S.; Bojsen-Møller, J.; Vissing, K. Muscle morphological and strength adaptations to endurance vs. Resistance training. J. Strength Cond. Res. 2012, 26, 398-407. [CrossRef] [PubMed] 
44. Ribeiro, A.S.; Avelar, A.; Schoenfeld, B.J.; Fleck, S.J.; Souza, M.F.; Padilha, C.S.; Cyrino, E.S. Analysis of the training load during a hypertrophy-type resistance training programme in men and women. Eur. J. Sport Sci. 2015, 15, 256-264. [CrossRef] [PubMed]

45. McBride, J.M.; Blaak, J.B.; Triplett-McBride, T. Effect of resistance exercise volume and complexity on emg, strength, and regional body composition. Eur. J. Appl. Physiol. 2003, 90, 626-632. [CrossRef] [PubMed]

46. Viitasalo, J.; Saukkonen, S.; Komi, P. Reproducibility of measurements of selected neuromuscular performance variables in man. Electromyogr. Clin. Neurophysiol. 1979, 20, 487-501.

47. Mitsiopoulos, N.; Baumgartner, R.; Heymsfield, S.; Lyons, W.; Gallagher, D.; Ross, R. Cadaver validation of skeletal muscle measurement by magnetic resonance imaging and computerized tomography. J. Appl. Physiol. 1998, 85, 115-122. [PubMed]

48. Heymsfield, S.; Smith, R.; Aulet, M.; Bensen, B.; Lichtman, S.; Wang, J.; Pierson, R.N. Appendicular skeletal muscle mass: Measurement by dual-photon absorptiometry. Am. J. Clin. Nutr. 1990, 52, 214-218. [PubMed]

49. Jacobson, J.A.; van Holsbeeck, M.T. Musculoskeletal ultrasonography. Orthop. Clin. N. Am. 1998, 29, $135-167$. [CrossRef]

50. Chhem, R.; Cardinal, E. Guidelines and Gamuts in Musculoskeletal Ultrasound; John Wiley \& Sons: Hoboken, NJ, USA, 1999.

51. De Maeseneer, M.; Marcelis, S.; Boulet, C.; Kichouh, M.; Shahabpour, M.; de Mey, J.; Cattrysse, E. Ultrasound of the knee with emphasis on the detailed anatomy of anterior, medial, and lateral structures. Skelet. Radiol. 2014, 43, 1025-1039. [CrossRef] [PubMed]

52. Seynnes, O.R.; de Boer, M.; Narici, M.V. Early skeletal muscle hypertrophy and architectural changes in response to high-intensity resistance training. J. Appl. Physiol. 2007, 102, 368-373. [CrossRef] [PubMed]

53. DeFreitas, J.M.; Beck, T.W.; Stock, M.S.; Dillon, M.A.; Kasishke, P.R. An examination of the time course of training-induced skeletal muscle hypertrophy. Eur. J. Appl. Physiol. 2011, 111, 2785-2790. [CrossRef] [PubMed]

54. Heymsfield, S.; Wang, Z.; Baumgartner, R.N.; Ross, R. Human body composition: Advances in models and methods. Annu. Rev. Nutr. 1997, 17, 527-558. [CrossRef] [PubMed]

55. Campos, G.E.; Luecke, T.J.; Wendeln, H.K.; Toma, K.; Hagerman, F.C.; Murray, T.F.; Ragg, K.E.; Ratamess, N.A.; Kraemer, W.J.; Staron, R.S. Muscular adaptations in response to three different resistance-training regimens: Specificity of repetition maximum training zones. Eur. J. Appl. Physiol. 2002, 88, 50-60. [CrossRef] [PubMed]

56. Tsarouhas, A.; Giakas, G.; Malizos, K.N.; Spiropoulos, G.; Sideris, V.; Koutedakis, Y.; Hantes, M.E. Dynamic effect of quadriceps muscle activation on anterior tibial translation after single-bundle and double-bundle anterior cruciate ligament reconstruction. Arthrosc. J. Arthrosc. Relat. Surg. 2015, 31, 1303-1309. [CrossRef] [PubMed]

57. Stojanovic, M.D.; Ostojic, S.M. Preventing acl injuries in team-sport athletes: A systematic review of training interventions. Res. Sports Med. 2012, 20, 223-238. [PubMed]

58. Herring, B. Responsive Kinetic Training Positively Increases Knee Joint Stability. Honors Thesis, The College at Brockport, Brockport, NY, USA, 5 September 2015.

59. Häkkinen, K.; Kraemer, W.J.; Newton, R.U.; Alen, M.; Häkkinen, M.; Kraemer, M.; Newton, M. Changes in electromyographic activity, muscle fibre and force production characteristics during heavy resistance/power strength training in middle-aged and older men and women. Acta Physiol. Scand. 2001, 171, 51-62. [CrossRef] [PubMed]

60. Markolf, K.L.; Graff-Radford, A.; Amstutz, H. In vivo knee stability. A quantitative assessment using an instrumented clinical testing apparatus. J. Bone Jt. Surg. Am. 1978, 60, 664-674. [PubMed]

61. Iversen, B.F.; Stürup, J.; Jacobsen, K.; Andersen, J. Implications of muscular defense in testing for the anterior drawer sign in the knee a stress radiographic investigation. Am. J. Sports Med. 1989, 17, 409-413. [CrossRef] [PubMed]

62. Daniel, D.M.; Malcom, L.L.; Losse, G.; Stone, M.L.; Sachs, R.; Burks, R. Instrumented measurement of anterior laxity of the knee. J. Bone Jt. Surg. Am. 1985, 67, 720-726. [PubMed]

63. Ekstrand, J.; Hägglund, M.; Waldén, M. Injury incidence and injury patterns in professional football: The uefa injury study. Br. J. Sports Med. 2011, 45, 553-558. [CrossRef] [PubMed] 
64. Prodromos, C.C.; Han, Y.; Rogowski, J.; Joyce, B.; Shi, K. A meta-analysis of the incidence of anterior cruciate ligament tears as a function of gender, sport, and a knee injury-reduction regimen. Arthroscopy 2007, 23, 1320-1325. [CrossRef] [PubMed]

65. Roi, G.S.; Nanni, G.; Tavana, R.; Tencone, F. Prevalence of anterior cruciate ligament reconstructions in professional soccer players. Sport Sci. Health 2006, 1, 118-121. [CrossRef]

66. Waldén, M.; Hägglund, M.; Werner, J.; Ekstrand, J. The epidemiology of anterior cruciate ligament injury in football (soccer): A review of the literature from a gender-related perspective. Knee Surg. Sports Traumatol. Arthrosc. 2011, 19, 3-10. [CrossRef] [PubMed]

(C) 2016 by the authors; licensee MDPI, Basel, Switzerland. This article is an open access article distributed under the terms and conditions of the Creative Commons Attribution (CC-BY) license (http://creativecommons.org/licenses/by/4.0/). 